\title{
Health Policy in Nepal: An Assessment of Clinical Guidelines in Relation to the Burden of Disease
}

\author{
Sara Hodgkinson ${ }^{1}$, Muhammad Saddiq², Julie Balen ${ }^{3}$ \\ ${ }^{1}$ BSocSc (Hons). MPH. PhD Scholar at Bankwest Curtin Economics Centre, Perth, Australia \\ ${ }^{2} \mathrm{MBBS}, \mathrm{MPH}, \mathrm{PhD}$. University teacher at University of Sheffield, UK \\ ${ }^{3} \mathrm{BSc}$ (Hons), PhD. Lecturer at University of Sheffield, UK
}

Corresponding Author : Sara Hodgkinson, sara.d.hodgkinson@gmail.com , +61 (0)892664353 (landline), +61 (0)435 913537 (mobile)

\section{Abstract}

Background: Clinical guidelines provide health workers with valuable information on how to diagnose and treat patients. In many low-income countries, there appears to be a lack of appropriate clinical guidelines for conditions with high rates of morbidity; Nepal is one such nation. It is useful to examine clinical guidelines in relation to disease burden to establish any gaps which might be addressed through changes in policy and practice.

Methods: The research adopted a mixed-methods approach, quantifying disease burden both nationally and in five districts served by PHASE Nepal - an NGO working in health and development - before contrasting this with available clinical guidelines in Nepal. Key informant interviews were subsequently conducted to gain greater insight into disease burden and guideline availability.

Results: Of the 10 diseases found to be most burdensome in the PHASE-served districts, 3 lacked any context-specific guidelines. NCDs were found to be prevalent at both district and national level, yet guideline provision for such conditions was minimal. Mental illness was highlighted by interview participants as a growing problem; however, Nepal does not currently have any utilisable clinical guidelines for mental health. Missing data was identified as a significant issue, with lack of mortality data hindering accurate quantification of local and national disease burden. 
Conclusions: There is evident need for more context-appropriate clinical guidelines in Nepal to effectively diagnose and treat diseases that are most burdensome within communities. Improving health data quality is fundamental to ensuring policy makers are equipped with accurate information from which to develop appropriate protocol.

Key Words: clinical guidelines; disease burden; health policy

\section{Introduction}

Clinical guidelines provide health workers with valuable diagnostic and treatment advice. It is particularly important for highly burdensome diseases to be addressed by comprehensive guidelines that are adaptable to different settings.

Recent research has highlighted the lack of appropriate guidelines in low-income countries..$^{1,2}$ Crucially, diseases with high rates of morbidity and mortality are not always addressed by appropriate clinical protocol. ${ }^{3}$ In Nepal, availability of up-to-date, context-specific guidelines has been called into question. ${ }^{4}$

The aim of this research is to examine clinical guidelines in Nepal in relation to disease burden to identify any gaps in guideline provision. The research was conducted in association with PHASE Worldwide and its partner NGO PHASE Nepal (hereafter referred to as 'PHASE'). Whilst offering insight into the national situation, the research focuses particularly on regions where PHASE provides services.

\section{Methods}

This mixed methods study consisting of analysis of secondary (quantitative) data and interviews sought to establish what clinical guidelines are available nationally in Nepal to examine how guidelines correlate with the burden of disease. Community level health data was obtained from PHASE for the period of Shrawan 2071 - Ashad 2072 (July 2014 - June 2015) and organised in Excel. Univariate analysis was conducted to reveal the diseases with highest collective morbidity across all 5 districts served by PHASE, namely, Bajura, Gorkha, Humla, Kavre and Sinhupalchok. The process was repeated for each of the individual districts to establish any differences in disease burden between them. National morbidity data from the Department of Health Services' most recent Annual Report 2070/71 (2013/14) ${ }^{5}$ was then analysed in the same way and subsequently compared with the PHASE data to establish any patterns or significant 
disparities. Ethical approval for this study was obtained from the University of Sheffield Research Ethics Committee, UK.

A comprehensive online search was executed to establish what context-specific clinical guidelines are currently available in Nepal. Key search terms were 'clinical guidelines', 'Nepal', and 'health policy'. All guideline documents located by the search were systematically examined to determine whether they contained diagnostic advice, treatment advice, or both. Documents were then organised in Excel and cross-checked with burden of disease figures to identify any gaps in clinical guideline provision. In addition, the clinical guideline manual produced and distributed by PHASE was examined to determine whether any current gaps in guideline provision are being met by this document.

A total of 5 semi-structured key informant $(\mathrm{KI})$ interviews were also conducted to gain a greater depth of insight into local disease burden. Convenience sampling was used to recruit participants from within PHASE's professional network. All interview participants were professionals with experience in - or links to - healthcare in Nepal. Interviews were conducted between February and March 2016, with informed verbal consent obtained from each participant prior to commencing. Interviews 1, 4 and 5 were conducted via Skype; interviews 2 and 3 were conducted in person. All interviews were semi-structured, were recorded on a smartphone and subsequently transcribed verbatim. Thematic analysis was then undertaken to identify any recurring themes that might substantiate or contradict the findings of the quantitative part of the study.

\section{Findings}

Analysis of a year's worth of morbidity data provided by PHASE revealed the collective disease burden in districts where the organisation works. Due to the differing topography and population structure of each of the 5 districts, it was useful to further analyse the data by district to expose any disparities in disease burden between regions. The 10 most burdensome diseases varied slightly from district to district - for example, whilst 9 of the 10 diseases with highest morbidity in Bajura were also the diseases found to have the highest collective morbidity across the 5 districts (see Table 1 and Figure 1), a number of other conditions not appearing in the collective 'top 10' contributed significantly to morbidity in Sindhupalchok. Similarly, some conditions found to be highly burdensome in terms of collective morbidity were less burdensome at individual 


\section{Original Article}

district level. Despite such disparities, the 10 diseases with highest collective morbidity all ultimately presented a substantial burden to each of the 5 districts.

Table 1. Diseases with highest collective morbidity across PHASE-served districts between July 2014 and June 2015, disaggregated by sex:

\begin{tabular}{|r|l|r|r|r|}
\hline RANK & NAME OF DISEASE & MALE CASES & FEMALE CASES & TOTAL CASES \\
\hline 1 & Gastritis & 3393 & 6408 & 9801 \\
\hline 2 & Upper Respiratory Tract Infection & 2477 & 2996 & 5473 \\
\hline 3 & ARI/Lower Respiratory Tract Infectior & 1775 & 2146 & 3921 \\
\hline 4 & Headache & 1415 & 2368 & 3783 \\
\hline 5 & Amoebic Dysentery & 1726 & 1938 & 3664 \\
\hline 6 & Falls/Injuries/Fractures & 2048 & 1399 & 3447 \\
\hline 7 & Acute Gastroenteritis & 1209 & 1246 & 2455 \\
\hline 8 & Conjunctivitis & 928 & 1303 & 2231 \\
\hline 9 & Arthritis & 808 & 1230 & 2038 \\
\hline 10 & Pneumonia & 951 & 882 & 1833 \\
\hline TOTAL & & 16730 & 21916 & 38646 \\
\hline & OTHER & 4323 & 5740 & 10063 \\
\hline
\end{tabular}

Figure 1. Diseases with highest collective morbidity across PHASE-served districts between July 2014 and June 2015, disaggregated by district:

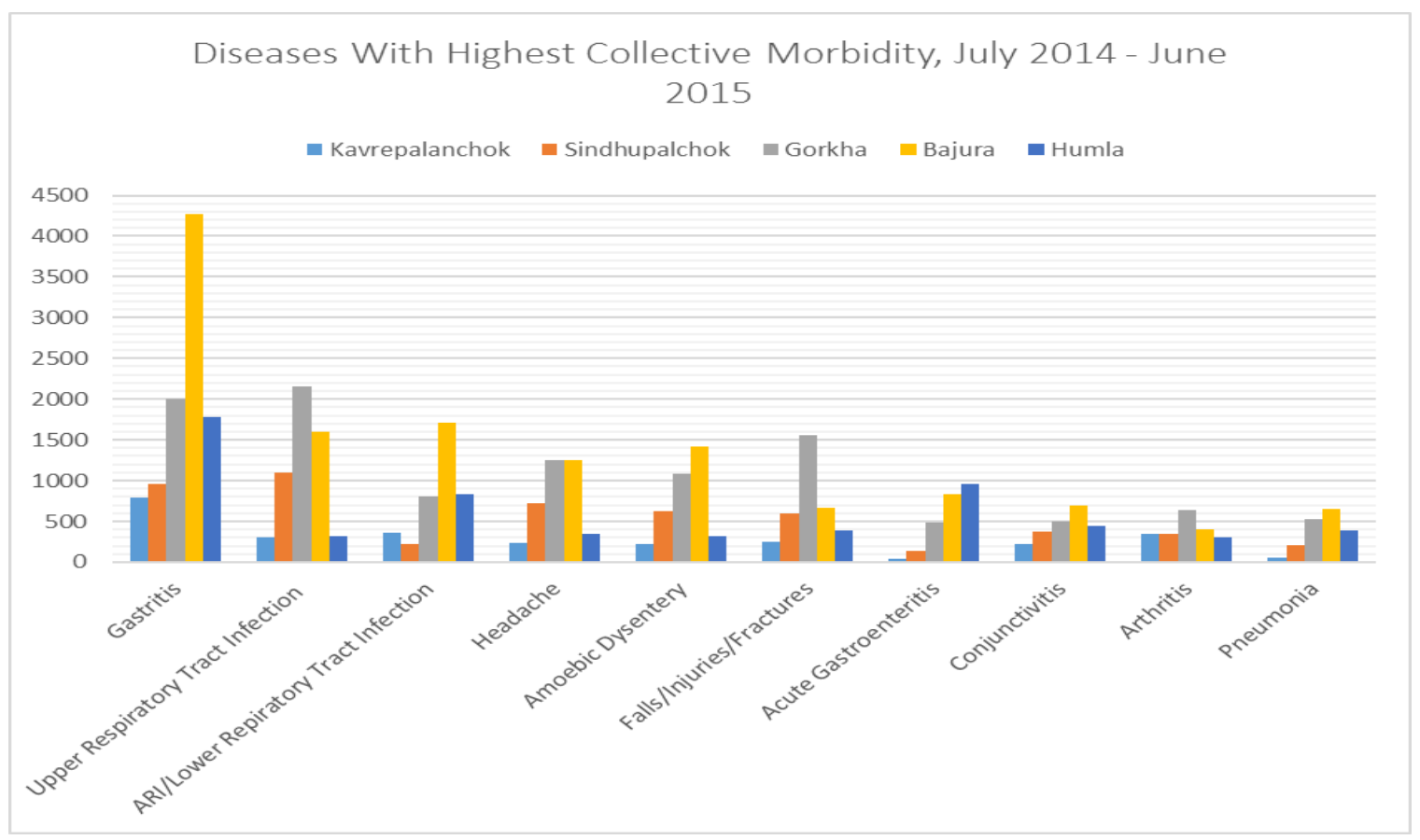


In addition to analysing health data collected by PHASE, publicly available datasets on morbidity were examined to establish the national burden of disease. This also facilitated identification of any differences between national morbidity rates and morbidity rates in the districts where PHASE works. Nepal's DoHS produces a yearly report in which a large amount of raw data on national morbidity is presented. As with the PHASE data, the DoHS Annual Report 2070/71 (2013/14) identified gastritis as the condition with the highest number of cases nationally (Table 2). Headache, upper respiratory tract infection and lower respiratory tract infection were - as in the PHASE-served districts - also found to be significant causes of morbidity. There were some notable divergences from the PHASE data, specifically the high burden of 'presumed non-infectious diarrhoea' and 'pyrexia of unknown origin' - conditions which, while present in the PHASE data, contributed a modest total of 871 and 884 cases respectively across the 5 districts. This discrepancy highlights the importance of guidelines on diagnostic criteria.

Table 2. Diseases with highest national morbidity, 2070/71 (2013/14):

\begin{tabular}{|r|l|r|r|}
\hline RANK & \multicolumn{1}{|c|}{ NAME OF DISEASE } & TOTAL CASES & \% OF NATIONAL MORBIDITY \\
\hline 1 & Gastritis & 1208863 & 5.58 \\
\hline 2 & Headache & 1112117 & 5.13 \\
\hline 3 & Upper Respiratory Tract Infection & 1099340 & 5.08 \\
\hline 4 & ARI/Lower Respiratory Tract Infectior & 1008898 & 4.66 \\
\hline 5 & Impetigo/Boils/Furunculosis & 744464 & 3.44 \\
\hline 6 & Intestinal Worms & 726221 & 3.35 \\
\hline 7 & Pyrexia of Unknown Origin & 674723 & 3.11 \\
\hline 8 & Presumed Non-Infectious Diarrhoea & 631996 & 2.92 \\
\hline 9 & Falls/Injuries/Fractures & 569128 & 2.63 \\
\hline 10 & Amoebic Dysentery & 537216 & 2.48 \\
\hline
\end{tabular}

A comprehensive literature search revealed the fragmented and under-developed nature of health policy in Nepal. Most of the guidelines located by the search were more than 5 years old. The level of detail varied considerably between guidelines - some documents provided comprehensive diagnostic advice and a complete treatment guide, whereas others merely offered a brief overview of symptoms and basic treatment advice. It remains unclear what the underlying cause of these discrepancies is. 
In terms of relevance to disease burden in the districts where PHASE works, the search found country-specific guidelines for only 7 of the 10 most burdensome conditions. Furthermore, whilst up-to-date information was found within the National Antibiotic Treatment Guidelines for gastritis, upper respiratory tract infection, lower respiratory tract infection, pneumonia and conjunctivitis, the document was brief in treatment guidance and offered minimal diagnostic advice for these conditions. Only 6 of the 10 diseases with highest national morbidity were addressed by current, context-specific clinical guidelines. For the diseases that did feature in documents with up-to-date advice, guidelines varied in content and depth and were rarely tailored to the Nepali context.

In addition, this study was provided with a copy of Simple Treatment Guidelines for Primary Health Care - a document compiled and published by PHASE in 2009, and distributed to health posts where PHASE staff work. In contrast to guidelines located by the online search, the PHASE document was found to provide comprehensive diagnostic and treatment advice for all diseases found to be most burdensome within the PHASE-served districts, and all but one of those most burdensome at country-level.

A number of themes emerged during analysis of interview data. A rise in non-communicable diseases was highlighted by all KIs. When asked specifically about the burden of disease in PHASE-served areas, one KI commented: "We still deal with diseases like diarrhoea... other water-borne diseases, they are still there. Recently there has been change in the disease pattern... more non-communicable diseases." (Interview 2.) Another KI indicated that NCDs such as COPD are perhaps becoming more visible as health NGOs expand into more remote communities (interview 1); it was also suggested that there is a growing gap between actual disease burden in rural Nepali communities and adequate government health provisions in these areas - an issue further compounded by staff absenteeism and resource constraints, as well as the additional challenges in health service delivery stemming from the 2015 earthquake. Mental health was also identified as an area in need of greater attention. The issue of undiagnosed and untreated mental health conditions in rural Nepali communities was raised by 4 of the $5 \mathrm{KIs}$. When asked to elaborate on mental health problems, one KI highlighted the issue of alcohol dependency, particularly among men (interview 4). The same $\mathrm{KI}$ also commented that, whilst there is presently minimal data available on the issue of mental health, communities 
appear to be experiencing a greater burden of mental health problems since the earthquake in April 2015. The absence of diagnostic tools and resources for the treatment of mental health problems was cited as a primary reason for mental health conditions remaining largely neglected in rural Nepali communities.

Lack of standardisation in healthcare was identified as a significant barrier to the effective monitoring, diagnosing and treatment of health complaints in Nepal. Despite the country boasting a "very well structured health care system on paper" (interview 5), availability of diagnostic equipment, of medical resources, of skilled health workers and of clinical guideline documents was said to vary considerably both between and within districts - consequently, the delivery of care within communities also varies (interviews 2 and 4). Health posts may have access to some government-issued clinical guidelines, and also to guidelines provided by NGOs such as PHASE, but KIs noted that inconsistencies in distribution remain. Interviews also revealed a lack of proper regulation of health workers to ensure that any guidelines that are available are effectively implemented.

All KIs commented on the difficulty of trusting official health figures given the lack of diagnostic capacity in some regions and nationwide inconsistencies in the reporting of health conditions. Missing data was cited as a key barrier to the development of appropriately focused clinical guidelines, along with lack of attention to context.

\section{Discussion}

The findings of this study offer several key insights into clinical guidelines and the burden of disease in Nepal. First, the significant presence of NCDs both at regional and national level contrasts starkly with the focus of appropriate clinical guidelines in Nepal. Both the PHASE data and the DoHS data identified a proliferation of NCDs, yet there appears to be minimal clinical guideline provision for such conditions. Interview participants corroborated the findings of the quantitative data analysis, commenting in detail on the rise in NCDs in regions where PHASE works; they also highlighted the lack of policy attention in this particular area. It has been suggested that NCD health policies possess the potential to have "a substantive impact on public health" in developing nations ${ }^{6}$ - thus to effectively tackle the most burdensome diseases in Nepal, NCDs must be addressed by policymakers with an urgency that matches the rate of proliferation. 
The second point to made in relation to burden of disease findings concerns the apparent absence of mental health problems from the health data. A total of 15 different mental health and neurology-related problems were listed in the PHASE morbidity report. There were just 8 recorded cases across all categories, despite the issue of mental health being raised on several occasions during $\mathrm{KI}$ interviews. This disparity between quantitative and qualitative data may be partly explained by the lack of mental health awareness in Nepal, both within communities and among local health workers. If individuals suffering from mental health problems are not aware of the symptoms of mental health - or of what services might be available - they are unlikely to seek help from clinical sources. The persistence of misinformation and stigma surrounding mental health may also prevent people from disclosing symptoms to those around them. Furthermore, if health workers are not adequately trained in mental health, they may not recognise symptoms in patients or be aware of how to help those in need. ${ }^{7,8}$ In each instance, cases of mental illness may go unreported and may thus be missed in health data.

A National Mental Health Policy is in existence in Nepal which is publicly available on the Ministry of Health website; however, the document is almost 20 years old and markedly brief. Moreover, there is little evidence to date of the document's implementation. This study was unable to locate any other context-specific clinical guidelines for mental health. In light of the findings of this study, and given the widespread psychological impact of the 2015 earthquake, it is critically important for mental health to receive greater policy attention. Distribution of comprehensive clinical guidelines with detailed diagnostic information and locally adaptable treatment advice may go some way towards addressing the growing problem of mental health in Nepal.

Whilst forthcoming with morbidity statistics and other useful information such as activity reports, PHASE was unable to provide mortality data because the information simply isn't available (interview 2). Country-level information on causes of death was also lacking, supporting Suvedi's claim that "data on the mortality pattern in Nepal in absolute terms are almost nonavailable". ${ }^{9}$ Without accurate information on mortality, raw health data cannot be effectively translated into comprehensible information via the use of tools such as the DALY, thus making it significantly more difficult to gauge the true impact of diseases and other health conditions on Nepali communities. This underscores the need for better recording and collection of health data nationwide in Nepal. 
This study revealed a scarcity of up-to-date, context-specific guidelines in Nepal. Of the 10 diseases found to be most burdensome in the PHASE-served districts, 3 conditions lacked any country-focused guidelines, whilst available context-specific guidelines for 5 of the other 7 conditions only offered nominal diagnostic and treatment advice. One of the biggest concerns among KIs was the lack of detailed, context-appropriate guidelines for the most burdensome conditions and diseases. This is a key area in need of attention from the Government of Nepal. Yet simply increasing the availability of context-appropriate guidelines would, by itself, be insufficient to address the issue. Graham and Harrison noted that a clinical guideline is only useful if it can be effectively implemented. ${ }^{10} \mathrm{It}$ is therefore important to ensure that any available guideline can be successfully utilised. This will require training of health workers and other staff as well as regular monitoring and evaluation.

Nepal is a nation of considerable diversity on many fronts, and this can present a number of challenges in terms of service delivery of any kind, including health. Diversity is something which needs to be reflected in any health policy or guideline to ensure that it can be adapted and effectively implemented regardless of location and resources. In addition, the training of health workers, as well as monitoring and evaluation activities, must also be improved to ensure that clinical guidelines are fully understood and appropriately utilised.

The focus of clinical guidelines in Nepal also needs to be addressed. Currently, the focus of context-specific guidelines in Nepal appears to correlate more closely with health issues prominent on the international agenda (such as HIVIAIDS and maternal health) than with diseases that are most prevalent among the Nepali population. Documents issued by NGOs and other private organisations may be filling a gap in guideline provision in Nepal. Yet such documents are often only circulated to staff within the organisations that have issued them, thus the impact of independently published guidelines is inevitably limited to certain areas and communities. Moreover, without any form of regulation, there is no way to effectively monitor the impact of privately published guidelines. To achieve true progress in public health in Nepal, all health workers must be equipped with comprehensive clinical guidelines for conditions that are widespread and significantly burdensome nationwide, and must be supported to implement them effectively.

\section{Conclusion}


In order to provide good quality health care, health workers need good guidelines, and in order to know what guidelines they need to produce, policy makers require accurate data. Improving the quality of health data is therefore fundamental to ensuring that policy makers are equipped with up-to-date and context-specific information from which to progress. Ensuring that health data effectively captures the health problems that are most prevalent within communities relies on the correct recording of diseases, which is, in turn, reliant on the capacity of health workers to properly identify and record symptoms and conditions. It is thus crucial to provide better training to improve the knowledge and diagnostic capacity of clinical staff throughout the country.

Better health education within communities may also be fundamental to improving health reporting and to ensuring that the most burdensome diseases gain appropriate attention at policy level. Equipping citizens with valuable health knowledge could provide individuals with greater control over their own health, and may also serve to decrease stigma around lessunderstood health issues such as mental illness - this could encourage people to seek treatment for conditions that may previously have been left untreated and thus unreported. Crucially, improving health education could increase public advocacy for significantly burdensome diseases presently overlooked by policy makers, such as NCDs. This may consequently increase political pressure on the Government of Nepal to provide more appropriately focused clinical guidelines.

Given the topography of Nepal and thus the isolated nature of many rural communities, together with the significant socioeconomic variance throughout the country, it is also essential to ensure that any nationally issued clinical guideline can be adapted to - and therefore utilised within any local context. This may necessitate greater government-led monitoring and evaluation of health and sub-health posts to observe and accordingly cater to the needs of different communities.

Each of these measures holds the potential to improve the correlation between clinical guidelines and disease burden in Nepal. Acting on any of the abovementioned recommendations could result in the issuance of more comprehensive and appropriately focused clinical guidelines which may ultimately improve the health of individuals in the districts where PHASE works, and throughout Nepal. 


\section{Original Article}

\section{Acknowledgements}

Several individuals at PHASE were central to the execution of this study. In particular, the researchers would like to thank Dr Gerda Pohl, Sarah Galvin, and Jiban Karki for their valuable input and assistance in getting this research off the ground.

Thanks must also go to the individuals who participated in interviews and provided researchers with a greater depth of insight into the study topic.

\section{References}

1. Goyet S, Barennes H, Libourel T, van Griensven J, Frutos R, Tarantola A. Knowledge translation: a case study on pneumonia research and clinical guidelines in a low-income country. Implement Sci 2014; 9 (82): 1-13. Available at:

http://implementationscience.biomedcentral.com/articles/10.1186/1748-5908-9-82

2. Puchalski Ritchie LM, Khan S, Moore JE, Timmings C, van Lettow M., Vogel JP, Khan DN, Mbaruku G, Mrisho M, Mugerwa K, Uka S, Metin Gülmezoglu A, Straus SE. Lowand middle-income countries face many common barriers to implementation of maternal health evidence products. J Clin Epidemiol 2016; 0 (0): 1-9. Available at:

https://www.ncbi.nlm.nih.gov/pubmed/26931284

3. Chakkalakal RJ, Cherlin E, Thompson J, Lindfield T, Lawson R, Bradley EH. Implementing clinical guidelines in low-income settings: a review of literature. Glob Public Health 2013; 8 (7): 784-795. Available at: https://www.ncbi.nlm.nih.gov/pubmed/23914758

4. Pohl G, Prasai R. Simple Treatment Guidelines for Primary Care. PHASE Nepal 2009

5. Department of Health Services. Annual report of the Department of Health Services 2070/71 (2013/2014). Kathmandu: Department of Health Services, Ministry of Health and Population (GoN) 2014

6. Mendis S. The policy agenda for prevention and control of non-communicable diseases. Br Med Bull 2010; 96 (1): 23-43. Available at: https://www.ncbi.nlm.nih.gov/pubmed/21059733 


\section{Original Article}

7. Luitel NP, Jordans MJD, Adhikari A, Upadhaya N, Hanlon C, Lund C, Komproe IH. Mental health care in Nepal: current situation and challenges for development of a district mental health care plan. Confl Health 2015; 9 (3): 1-11. Available at: https://www.ncbi.nlm.nih.gov/pmc/articles/PMC4331482/

8. van Teijlingen E, Simkhada P, Devkota B, Fanning P, Ireland J, Simkhada B, Sherchan L, Chandra Silwal R, Pradhan S, Maharjan SK, Maharjan RK. Mental Health Issues in Pregnant Women in Nepal. Nepal J Epidemiol 2015; 5 (3): 499-501. Available at: https://www.ncbi.nlm.nih.gov/pmc/articles/PMC4762507/

9. Suvedi BK. Of what diseases are Nepalese people dying? Kathmandu Univ Med J 2007; 5 (1; 17): 121-123. Available at: www.kumj.com.np/issue/17/121-123.pdf

10. Graham ID, Harrison MB. EBN users' guide: Evaluation and adaptation of clinical practice guidelines. Evid Based Nurs 2005; 8: 68-72 